\title{
The Effects of Parental Beliefs and Expectation on the Perception of Compliance in Adolescents
}

\author{
Anton James
}

\begin{abstract}
Noncompliance accounts for the majority of referrals to psychologists in the US, and psychologists tend to put more emphasis on information provided by parents rather than their children. However, parents' perception of noncompliance in their children is influenced by their personal notions of how a child should ideally behave, as well as their expectations of their children's behaviour. Clinical complexity associated with diagnosis of disorders related noncompliance and the biased perception by parents, may lead to misdiagnosis of ODD and CD amongst adolescents.
\end{abstract}

Index Terms-Conduct disorder, compliance, clinical complexity, misdiagnosis, and oppositional defiant disorder.

\section{INTRODUCTION}

Noncompliance of children has been a major concern for teachers and parents as it is seen as an obstacle to their academic advancement and safety. Adults complain that noncompliance is an important and common problem with children [1]-[3]. This has led to noncompliance in children accounting for the majority of referrals to psychologists by adults in the US [4], [5]; with the prevalent rate of noncompliance, in clinical samples, ranging from $65 \%$ to $92 \%$ [6]. Moreover, severe noncompliance is one of the criteria for a child being diagnosed as having ODD (oppositional defiant disorder), CD (conduct disorder) [4] 'attention deficit hyperactivity disorder' (ADHD), mood disorders, psychotic disorders, autistic disorder [7], or being misdiagnosed as severely mentally handicapped [8]. This calls for a scrutiny of the perception of children's noncompliance by parents.

\section{BODY}

\section{A. What is Compliance?}

Compliance in children has largely been studied in the behaviourist tradition, which follows a positivist paradigm; therefore, an emphasis on objective observation is pivotal to its definition. Currently used definitions of compliance can be traced back to Forehand, who summarised pre-1977 studies and concluded that compliance can be defined in terms of latency and/or duration [9]. Latency refers to the time elapsed between asking the child to perform a task and the initiation of that task by that child; duration refers to the total time taken to complete the requested task. Since Forehand's review, researchers investigating compliance in

Manuscript received March 18, 2013; revised May 23, 2013.

Anton James is with the University of Manchester, UK (e-mail: antonuoft@yahoo.com). the behaviourist tradition, consistently continue to define it in these terms; for example, "...compliance [is] defined as the participant initiating the response specified by the instruction within 10 [seconds] of the instruction being delivered" [10]. Subsequently such definitions have also been adopted by the non-behaviourist tradition [11].

However, the above approach to defining compliance is flawed on multiple levels. Firstly, it leads to the exclusion of certain behaviours from the construct of compliance; for instance duration and latency are not appropriate measures for smoking when the sign in the school says 'do not smoke' or defying a parental demand to abstain from premarital sex. Secondly, definitions with predetermined standards are primarily concerned with the child obeying the instructions of an adult, which is a practitioner-centeredness problem [12]. A certain level of refusal behaviours in children are adaptive and functional [13] and in some cases total refusal behaviour is completely acceptable. Finally, on a cognitive level, it is problematic for an arbitrary person to categorise behaviour as compliant or noncompliant using arbitrary criteria. Refusal or uncooperative behaviours can take several forms, such as breaking a curfew or failing to do homework; each parent assigns a different value for each refusal or uncooperative behaviour, which may be socioculturally shaped [14]. For example, parents from a certain culture may be more concerned about their daughter 'breaking the curfew' than 'failing to do homework'. Therefore, any arbitrary criteria by a third party places these definitions outside the parameters of parental cognition. A compliant and noncompliant behaviour is based on the perspective and beliefs of adults, which vary from person to person.

\section{B. Family Related Cognitions}

Family related cognitions have been explored and defined at various levels. Bugental and Johnston summarised them as: belonging to 1) The way things should be, ideally, in a family (evaluative-descriptive cognition); 2) The way things are perceived in a family (descriptive cognition); and 3 ) Convergence or divergence from the way things are and the way things should be in a family (efficacy cognitions) [15]. The parental beliefs on ideal levels of compliance, in adolescents, and their level of expectation for their adolescent is a form of evaluative-descriptive cognition. The parental perception of levels of compliance in their children is a type of descriptive cognition.

Parents' belief about what should be ideal levels of compliance for their adolescent children varies between parents [16], [17]. This directly impacts upon the behavioural expectations they have of their children and these expectations are generated as a result of these beliefs 
[18]. Sometimes children modify their compliance level according to parental expectations [19]. When expectation and level of compliance converge, parents are less likely to perceive their children's behaviour as problematic. On the hand, when the level of compliance does not meet their expectation, parents perceive those behaviours to be more problematic [7]. This convergence and divergence of ideal and perceived, is the third type of cognition (i.e. efficacy cognition) that was described earlier. Consistent with this view, research has shown that parental expectation affects how they perceive their children's behaviour as problematic [7], [16]. Kalb and Loeber also argue that perception of how problematic these behaviours are (descriptive cognitions), in turn influences their perception of the severity or frequency of noncompliance (descriptive cognitions) [2]. Therefore, they argue that these perceptions do not reflect the child's actual behaviour(s); rather it will be influenced by their perception of how problematic those behaviours are. This perception of frequency or severity of noncompliance plays a crucial role when assessing children's noncompliance.

\section{Effects on Assessment of Noncompliance}

According to DSM-4-TR, noncompliance is one of the diagnostic criteria for $\mathrm{CD}$ and ODD, which is measured in terms of frequency and severity [20]. For instance, one of the diagnostic criteria for ODD is that the child "often [frequency] actively defies or refuses to comply with adults' requests or rules" (p.102). Also, the DSM-4-TR uses similar subjective wording in diagnostic criteria for $\mathrm{CD}$, such as, the child 'has deliberately [severity] destroyed others' property', [is] 'often [frequency] breaking the curfew', or [is] 'often [frequency] truant from school' (p. 99). Therefore, psychologists often use subjective assessment methods to evaluate children's noncompliance [21].

This subjective measurement could amount to what is known as 'clinical complexity' [22] when assessing CD and ODD, due to variation in evaluative-descriptive cognition:

1) Parental belief of ideal levels of compliance and their expectations (evaluative-descriptive cognitions) vary from parent to parent [2], [17], [23]. Therefore, parental perception of severity or frequency of noncompliance (descriptive cognition) would also vary, without truly reflecting a child's actual noncompliance. For example, conduct problems in children are more common among those whose parents have biased beliefs and expectations about children's behaviour [7].

2) Any bias in parental descriptive cognitions has serious implications in assessment of noncompliance, because the psychologists may misunderstand the extent of noncompliance. This is due to psychologists inclining to agree more with parents than children about children's problematic behaviour [24].

3) Further, parents are seen as advocates for access to referred services for their children in England, where diagnosis for CD and ODD are made [25].

This clinical complexity, particularly in relation to potential bias in descriptive cognitions, may lead to children being misdiagnosed with $\mathrm{CD}$ or ODD, as shown in Fig. 1 below:

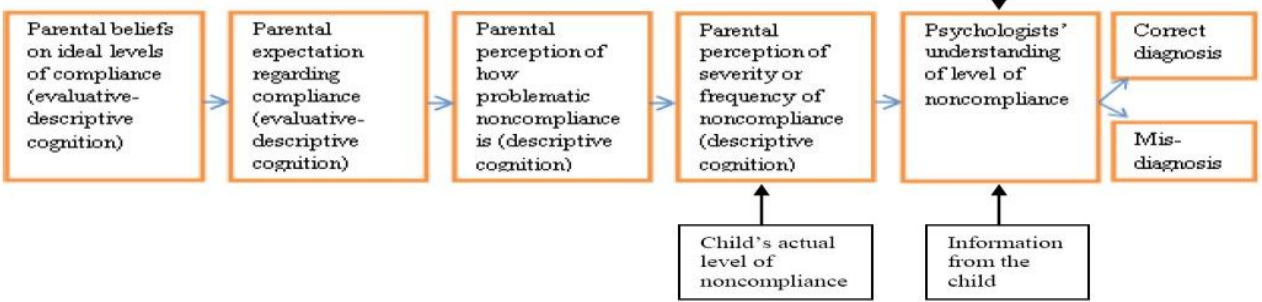

Fig. 1. The effect of beliefs and expectation on psychologists' understanding of noncompliance

\section{Adolescence}

The milestone of adolescent development is accentuated not only by physical and cognitive maturity but also by the development of 'self' [26]. For instance, Smetana et al. indicate that adolescence begins in biology and ends in culture [27]. These developments prepare adolescents for 'autonomy', which is essential for adaptive psychological functioning [28]. This is a challenge for parents, who have to revise the rules and levels of noncompliance to accommodate the development of such autonomy [29]. They may also be annoyed that cognitive maturity, during this period, allows adolescents to skilfully win arguments over parents [30]. These new developments could be interpreted as defying parents' expectations of their children, in complying with requests and rules, when they are hesitant to accommodate their child's autonomy during adolescence [31]-[33]. For example, Collins found a higher discrepancy between parents' perception of ideal and perceived behaviour among children who are going through the transition to adolescence, in comparison to younger or older children [34]. This discrepancy may be due to the fact that parents continue to expect adolescents to comply as younger children, even though adolescents are going through a stage of acquiring autonomy [29].

\section{E. Parental Knowledge}

One of the aspects of 'parental knowledge' refers to parenting skills and the extent to which parents understand developmental norms, milestones and the process of child development [35]. This is one of the variables that may affect their belief about an ideal level of compliance. Ehrensaft et al. suggest that "...if parental complaints reflect not only the child's behavio[u]r, but also the parent's appraisal of the behavio[u]r in relation to the child's developmental level, we may expect these to decrease from pre-adolescence to adolescence and emerging adulthood" [36]. However, scholars agree that parental complaints tend 
to increase when their children move into adolescence [37][40]. There is substantial research dedicated to particular aspects of parental knowledge, such as parenting skills [41]. However, there is a severe paucity of research in other aspects of parental knowledge, such as parents' knowledge of adolescence development.

\section{CONCLUSION}

The diagnosis of $\mathrm{CD}$ is higher at the onset of adolescence in the US [42] as well as in the UK [43] and coincides with an increase in parental complaints about adolescents. The DSM-4-TR goes on to identify 'adolescence onset type' as a separate category of $\mathrm{CD}$ [20]. It is feasible that the rise in diagnoses of conduct related disorders is due to clinical complexity, which is associated with biased parental beliefs and parental expectation of compliance being inconsistent with adolescent development. Due to such clinical complexity, psychologists should proceed with caution when diagnosing $\mathrm{CD}$ and ODD among adolescents. Information could be collected from various sources rather than primarily relying on parents. This may help to minimise clinical complexity. There appears to be a paucity of research in this area. Therefore, further research is needed to directly measure the effect that parental beliefs and expectation have on their perception of compliance in their adolescent children.

\section{ACKNOWLEDGMENT}

I thank $\mathrm{Su}$ Corcoran and Cecil Nielan for their contribution in editing this paper.

\section{REFERENCES}

[1] P. S. Strand, R. G. Wahler, and M. Herring, "Momentum in child compliance and opposition," Journal of Child and Family Studies, vol. 9, no. 3, pp. 363-375, 2000.

[2] L. M. Kalb and R. Loeber, "Child disobedience and noncompliance: A review," Pediatrics, vol. 111, no. 3, pp. 641-652, 2003

[3] K. A. Feinfield and B. L. Baker, "Empirical support for a treatment program for families of young children with externalizing problems," Journal of Clinical Child and Adolescent Psychology, vol. 33, no. 1, pp. 182-195, 2004.

[4] M. Hakman and M. Sullivan, "The effect of task and maternal verbosity on compliance in toddlers," Infant and Child Development, vol. 18 , no. 2, pp. 195-205, 2009.

[5] R. J. McMahon, R. L. Forehand, and S. L. Foster, Helping the Noncompliant Child: Family-based Treatment for Oppositional Behavior, New York: The Guilford Press, 2005.

[6] T. M. Achenbach and C. S. Edelbrock, Manual for the Child Behavior Checklist and Revised Child Behavior Profile, Burlington University of Vermont, 1983.

[7] R. L. McMahon and R. L. Forehand, Helping the Noncompliant Child: Family-based Treatment for Oppositional Behavior, London: The Guilford Press, 2005.

[8] A. Alborz, "Parental Input in Education: An Illustration," Journal of the British Institute of Mental Handicap (APEX), vol. 21, no. 4, pp. 142-146, 1993.

[9] R. Forehand, "Child compliance to parental requests: Behavioral analysis and treatment, in progress" in Behavior Modification, M. Hersen, R. Eisler, and P. Miller, Eds, Academic Press: London, 1977, pp. 111-148.

[10] M. P. Normand, K. Kestner, and J. Jessel, "An analysis of stimuli that influence compliance during the high-probability instruction sequence," Journal of Applied Behavior Analysis, vol. 43. no. 4, pp. 735-738, 2010.

[11] J. A. A. Abe and C. E. Izard, "Compliance, noncompliance strategies, and the correlates of compliance in 5-year-old Japanese and American children," Social Development, vol. 8, no. 1, pp. 1-20, 1999.
[12] P. Karoly, "Enlarging the scope of the compliance construct: Toward developmental and motivational relevance," in Developmental Aspects of Health Compliance, N. Krasnegor et al., Eds. Lawrence Erlbaum Associates: London, 1993, pp. 11-28.

[13] K. M. Stephenson and G. P. Hanley, "Preschoolers' compliance with simple instructions: A descriptive and experimental evaluation," Journal of Applied Behavior Analysis, vol. 43, no. 2, pp. 229-247, 2010.

[14] A. L. Dlott et al., "Commands, competence, and carino: Maternal socialization practices in Mexican American families," Developmental Psychology, vol. 46, no. 3, pp. 566-578, 2010.

[15] D. B. Bugental and C. Johnston, "Parental and child cognitions in the context of the family," Annual Review of Psychology, vol. 51, pp. 315-344, 2000.

[16] C. N. Buchanan and G. N. Holmbeck, "Measuring beliefs about adolescent personality and behavior," Journal of Youth and Adolescence, vol. 27, no. 5, pp. 607-627, 1998.

[17] B. M. Gfellner, "Culture and consistency in ideal and actual childrearing practices: A study of Canadian Indian and White parents," Journal of Comparative Family Studies, vol. 21, no. 3, pp. 413-423, 1990.

[18] R. Casati and E. Pasquinelli, "How can you be surprised? The case for volatile expectations," Phenomenology and the Cognitive Sciences, vol. 6, no. 1-2, pp. 171-183, 2007.

[19] J. Li et al., "Why attend school? Chinese immigrant and European American preschoolers' views and outcomes," Developmental Psychology, vol. 46, no. 6, pp. 1637-1650, 2010.

[20] APA, Diagnostic and Statistical Manual of Mental Disorders, Text Revision, fourth edition. 4 ed. Washington, DC: American Psychiatric Association, 2000.

[21] A. C. W. Bueno, B. C. dos Santos, and C. B. de Moura, "Child compliance: Definitions, behavioral measurement and research results," Psicologia: Teoria e Pesquisa, vol. 26, no. 2, pp. 203-216, 2010.

[22] J. E. Mezzich and I. M. Salloum, "Clinical complexity and personcentered integrative diagnosis," World Psychiatry, vol. 7, no. 1, pp. 1, 2008.

[23] A. L. Koenig, D. Cicchetti, and F. A. Rogosch, "Child compliance/noncompliance and maternal contributors to internalization in maltreating and nonmaltreating dyads," Child Development, vol. 71, no. 4, pp. 1018-1032, 2000.

[24] K. Hawley and J. R. Weisz, "Child, parent, and therapist (Dis) agreement on target problems in outpatient therapy: The therapist's dilemma and its implications," Journal of Consulting and Clinical Psychology, vol. 71, no. 1, pp. 62-70, 2003

[25] K. Sayal et al., "Pathways to care in children at risk of attentiondeficit hyperactivity disorder," British Journal of Psychiatry, vol. 181. no. 1 , pp. $43-48,2002$.

[26] L. Steinberg and A. S. Morris, "Adolescent development," Annиal Review of Psychology, vol. 52, pp. 83-110, 2001.

[27] J. G. Smetana, N. C. Barr, and A. Metzger, "Adolescent development in interpersonal and societal contexts," Annual Review of Psychology, vol. 57, pp. 255-284, 2006

[28] S. Petegem et al., "On the association between adolescent autonomy and psychosocial functioning: Examining decisional independence from a self-determination theory perspective," Developmental Psychology, vol. 48, no. 1, pp. 76-88, 2012.

[29] G. N. Holmbeck, R. L. Paikoff, and J. B. Gunn, "Parenting adolescents," in Handbook of Parenting, Children and parenting, M. H. Bornstein, Ed. Lawrence Erlbaum Associates: Hove, vol. 1, 1995, pp. 91-118.

[30] L. Hendry and M. Kloep, "Adolescence and adulthood: Transitions and transformations Palgrave insights," in Psychology, N. Holt and R. Lewis, Eds, New York: Palgrave Macmillan, 2012.

[31] M. Fleming, "Adolescent autonomy: Desire, achievement and disobeying parents between early and late adolescence," Australian Journal of Education and Developmental Psychology, vol. 5, pp. 1-16, 2005.

[32] J. S. Phinney et al., "Autonomy and relatedness in adolescent-parent disagreements: Ethnic and developmental factors," Journal of Adolescent Research, vol. 20, no. 1, pp. 8-39, 2005

[33] L. Steinberg and J. S. Silk, "Parenting adolescents," in Handbook of Parenting, Children and Parenting, M. H. Bornstein, Ed. Lawrence Erlbaum Associates Publishers, US: Mahwah, NJ, vol. 1, 2002, pp. $103-133$

[34] W. A. Collins, "Parent-child relationships in the transition to adolescence: Continuity and change in interaction, affect, and cognition," in From Childhood to Adolescence: A Transitional Period? R. Montemayor, G. Adams, and T. Gullotta, Eds. Sage Publications: Thousand Oaks, CA. 1990, pp. 85-106. 
[35] A. A. Benasich and J. B. Gunn, "Maternal attitudes and knowledge of child-rearing: Associations with family and child outcomes," Child Development, vol. 67, no. 3, pp. 1186-1205, 1996.

[36] M. K. Ehrensaft et al., "Development transitions in youth behavioral opposition and maternal beliefs in social ecological context," Journal of Child and Family Studies, vol. 16, no. 4, pp. 577-588, 2007.

[37] K. Burke, L. Brennan, and S. Roney, "A randomized controlled trial of the efficacy of the ABCD: Parenting Young Adolescents Program: Rationale and methodology," Child and Adolescent Psychiatry and Mental Health, vol. 4, no. 22, pp. 1-14, 2010.

[38] L. Shanahan et al., "Conflict frequency with mothers and fathers from middle childhood to late adolescence: Within and between-families comparisons," Developmental Psychology, vol. 43, no. 3, pp. 539-550 2007.

[39] C. L. Shearer, A. C. Crouter, and S. M. McHale, "Parents' Perceptions of Changes in Mother-Child and Father-Child Relationships During Adolescence," Journal of Adolescent Research, vol. 20, no. 6, pp. 662-684, 2005.

[40] K. Weichold, S. Buttig, and R. K. Silbereisen, "Effects of pubertal timing on communication behaviors and stress reactivity in young women during conflict discussions with their mothers," Journal of Youth and Adolescence, vol. 37, no. 9, pp. 1123-1133, 2008.

[41] M. A. Lippold, M. T. Greenberg, and M. E. Feinberg, "A dyadic approach to understanding the relationship of maternal knowledge of youths' activities to youths' problem behavior among rural adolescents," Journal of Youth and Adolescence, vol. 40, no. 9, pp. 1178-1191, 2011.

[42] B. Maughan et al., "Conduct disorder and oppositional defiant disorder in a national sample: Developmental epidemiology," Journal of Child Psychology and Psychiatry, vol. 45, no. 3, pp. 609-621, 2004.

[43] NICE, Parent-Training/Education Programmers in the Management of Children with Conduct Disorders, National Institute for Health and Clinical Excellence: London, 2006.

Anton James was born in Sri Lanka in 1979, and currently is a PhD candidate at the University of Manchester, UK. Degrees obtained: BSc in 'psychology' (2003) at University of Toronto, Canada. Post graduate diploma in 'special education' (2006) at the Penn State University, USA. Post graduate diploma in 'Professional studies in education' (2009) at the Open University, UK. Master of Education in 'Equality and diversity' (2009) at the Open university, UK. Master of Science in 'research in Education' (2012) at the University Of Manchester, UK.

Mr. James currently works as a behavior analyst, specializing in children with behavioral problems. He is a 'Board Certified Behavior Analyst' from the 'Behavior Analyst Certification Board' in the USA. He is also a member of the British and Irish psychological societies. 